\title{
Nonlinear Frequency Response of Comb-Driven Microscanners
}

\author{
Caglar Ataman, Hakan Urey \\ Optical Microsystems Laboratory, Department of Electrical Engineering, Koc University \\ Rumeli Feneri Yolu, 34450, Sariyer, Istanbul, TURKEY \\ Phone: +90-212-338-1474, E-mail: cataman@ku.edu.tr, hurey@ku.edu.tr
}

\begin{abstract}
Accurate prediction of the dynamic behavior of comb-driven MEMS microscanners is important to optimize the actuator and structure design. In this paper, a numerical and an analytical model for the dynamic analysis of comb-driven microscanners under different excitation schemes are presented. The numerical model is based on a second order nonlinear differential equation. Due to the nature of the torque function, this governing equation of motion is a parametric nonlinear ODE, which exhibits hysteretic frequency domain behavior and subharmonic oscillations. . Experimental results and approximate analytical expressions for this nonlinear torque function of the comb-drive are presented. Amplitude and phase relationship between the excitation signal and the resultant oscillations at different excitation frequencies are measured and we show that they are in close agreement with the numerical simulations. Analytical model uses perturbation methods to reach approximate close-form expressions for the dynamic behavior of the device in the first parametric resonance region. It is also utilized to predict the stability regions on the frequencyexcitation voltage plane, where the device exhibit hysterical characteristics. Analytical and numerical modeling approaches proposed in this paper provides a simple yet powerful way to analyze the nonlinear frequency response of comb-driven actuators and simplify the design process for a microscanner based system.
\end{abstract}

Keywords: Comb-drive actuation, microscanner, subharmonic oscillation, parametric resonance

\section{INTRODUCTION}

Comb-driven electrostatic actuation is widely used in various microstructures. Comb structures greatly enhance the net capacitance variation and therefore, high actuation forces can be reached with relatively low drive voltages ${ }^{1}$. Some applications that utilize comb-drive structures include microscanner ${ }^{2,3}$, mechanical filters ${ }^{4,5}$, and optical switches ${ }^{6,7}$.

In-plane motion of a comb drive is governed by a second order constant-coefficient differential equation. The resulting system is a Simple Harmonic Oscillator (SHO). In torsional mode, the motion is out-of-plane. Actuation force is a nonlinear function of angular displacement and time. Therefore, the governing second order differential equation (DE) is no longer a constant coefficient linear DE, but a nonlinear DE with time-varying coefficients. Time varying coefficients in the governing equation lead to time dependant torsional stiffness, and the governing DE defines a parametric system that exhibits hysterical frequency response, generates subharmonic oscillations and has multiple orders of parametric resonances. Parametric oscillation is not a rare phenomenon in engineering, and is investigated in great detail, elsewhere ${ }^{8,9}$. Turner developed analytical formulas for a comb-driven resonator used for scanning tunneling microscopy ${ }^{10,11}$, but this is the first time that such an analysis is carried out for a microscanner. We also developed accurate numerical simulations to study the parametric resonances for arbitrary excitation waveforms.

We first discuss the structure and the typical frequency characteristics of the comb-driven microscanners used in this study. The remainder of the paper has three parts where we present our results. In section 3, we propose a numerical model for the device and compare the simulation results with experimental data. In section 4, an analytical solution of the equation of motion near the first parametric resonance is presented. Section 5 presents further experimental data on the frequency response of the device for different excitation waveforms. The last section gives an insight for future work in this area. 


\section{DEVICE STRUCTURE AND DYNAMIC CHARACTERISTICS}

The device that we have investigated in this work is a $1 \mathrm{D}$, single-crystalline silicon comb-actuated microscanner. The structure is consisted of a movable, aluminum covered mirror, a fixed frame and two flexure beams that bind the mirror to the frame. The flexures act as torsional springs that pull the mirror back to its rest position. The mirror is $2 \mathrm{~mm}$ long, $2 \mathrm{~mm}$ wide and $30 \mu \mathrm{m}$ thick. The flexure dimensions are $550 \mu \mathrm{m}$ in length, $10 \mu \mathrm{m}$ in width and $30 \mu \mathrm{m}$ in thickness. Both the comb fingers and the gap between the comb fingers are $5 \mu \mathrm{m}$ wide. Comb fingers are placed on both sides of the mirror. Mirror and the frame are electrostatically isolated with isolation trenches, so that different potentials can be applied to the fixed and movable comb fingers. The device was fabricated by Fraunhofer Institute for Photonic Microsystems (IPMS), in Dresden-Germany. More information on the design and fabrication of the device can be found in the related works by Fraunhofer IPMS ${ }^{2,12,13}$. Simple sketch of the device and micrograph of the comb fingers are shown below.

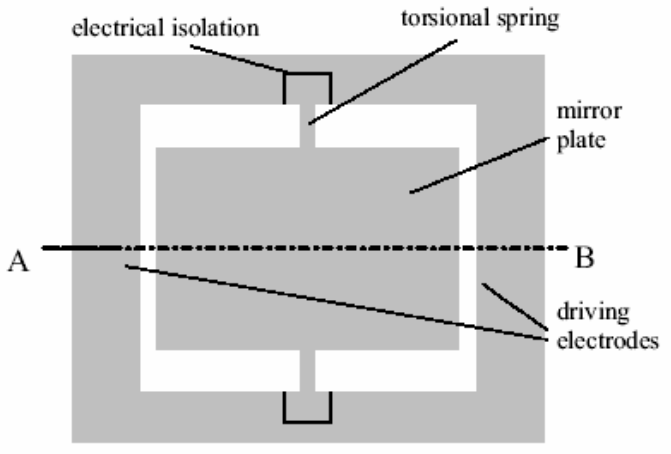

Figure 1: General Device Layout ${ }^{12}$

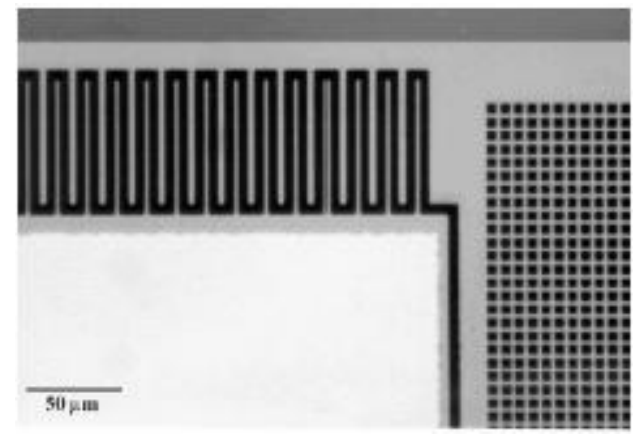

Figure 2: Micrograph of the comb fingers ${ }^{12}$

Biaxial microscanners that produce a 2-D raster pattern with comb-drive actuation are also available. In 2D mirror structure, there is a reflective mirror in the middle, a movable inner frame and a fixed outer frame. Innermost mirror and the movable frame have comb-drives in perpendicular orientation; therefore, the device can manage 2D scanning. Since the actuation mechanism is exactly the same, dynamic characteristics of $1 \mathrm{D}$ and $2 \mathrm{D}$ scanners are the same. Figure 1 shows the frequency response curves for horizontal and vertical axis of a 2D microscanner. The shape of the response curve is typical for all similar devices. Frequency response is nonlinear and shows hysterical behavior. The path traced on the $\theta-f$ curve when frequency is increased quasi-statically from a lover frequency is different than the case, in which the frequency is swept down from a higher frequency.

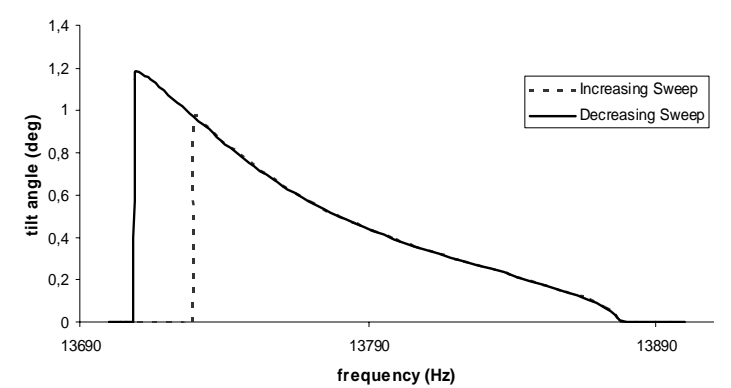

(a)

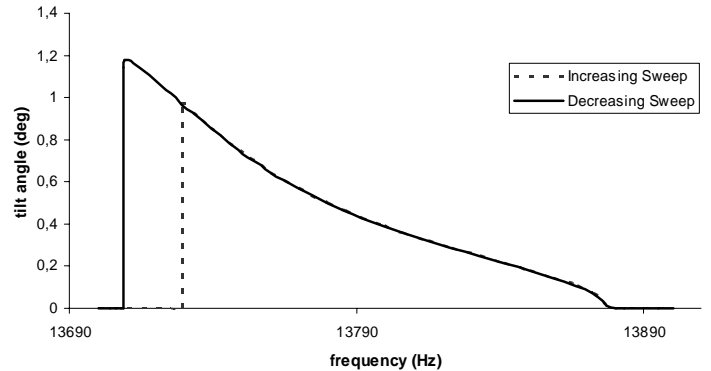

(b)

Figure 3: Frequency response of a) vertical, b) horizontal axis of a 2D comb-driven microstructure 


\section{SYSTEM MODELING AND NUMERICAL ANALYSIS}

Comb-actuated micromirror devices exhibit a hysterical behavior and generate subharmonic oscillations. Such phenomena are typical for nonlinear parametric systems. In order to have a parametric equation of motion for our microscanner, electrical or torsional stiffness of the device should be time dependant. It is known that the general equation of motion for a microscanner can be written as follows ${ }^{14}$ :

$$
I_{m} \frac{d^{2} \theta}{d t^{2}}+b \frac{d \theta}{d t}+K_{f} \theta=M(\theta)
$$

where,
$I_{m}$ : Mass moment of inertia;
$b$ : Damping constant;
$K_{f}$ : Torsional stiffness;
$M(\theta)$ : Torque function.

Amount of torque produced by the comb actuators as a function of the tilt angle is given as:

$$
M(\theta)=2 N \frac{1}{2} \frac{d C}{d \theta} V(t)^{2}
$$

where, $N$ is the number of fingers on one side of the mirror. $M(\theta)$ is proportional to the rate of change of the energy stored in the actuator combs with respect to $\theta$. Change in the stored energy is proportional to the change in the total capacitance of the comb actuators, which is proportional to the overlap area of comb fingers. Overlap area between the finger plates is a piecewise continuous function with three different regions. This also leads, when the fringing effects are ignored, to a piecewise continuous torque function. If the comb fingers on different frames are designed to be coplanar without any vertical offset in between and have the same thickness, the forcing function becomes an even function. If the structure were perfectly symmetrical, oscillations would not start, but there is always some asymmetry due to processing and even small force is sufficient to get the oscillations started. When fringing fields are ignored, the rate of change of total capacitance with tilt angle can be found by expressing the overlap area of the comb fingers as a function of $\theta$. The resultant function is piecewise and can be expressed as:

$$
\begin{aligned}
& \text { Case I (when } \left.\theta<\frac{t_{m}}{\frac{D}{2}+f_{l}}\right) \\
& \frac{d C}{d \theta}=-\frac{3 \varepsilon}{2 g}\left(\frac{D}{2}+f_{l}\right)^{2} \theta^{2}+\frac{2 \varepsilon}{g} t_{m}\left(\frac{D}{2}+f_{l}\right) \theta+\frac{\varepsilon}{g}\left(\frac{x_{0}{ }^{2}}{2}-\left(\frac{D}{2}+f_{l}\right) x_{0}-\frac{t_{m}{ }^{2}}{2}\right)-\varepsilon \frac{\left(\frac{D}{2}+f_{l}\right)}{\left(f_{l}-x_{0}\right)} f_{d}
\end{aligned}
$$

Case II (when $\frac{t_{m}}{\frac{D}{2}+f_{l}}<\theta<\frac{t_{m}}{\frac{D}{2}+f_{l}-x_{0}}$

$$
\frac{d C}{d \theta}=\frac{\varepsilon}{2 g} x_{0}\left(\frac{D}{2}+f_{l}-x_{0}\right)-\frac{\varepsilon t_{m}{ }^{2}}{2 g \theta}
$$

Case III (when $\theta>\frac{t_{m}}{\frac{D}{2}+f_{l}-x_{0}}$ )

$$
\frac{d C}{d \theta}=0
$$


where,

$D$ : Mirror Width

$f_{l}$ : Finger Length

g: Gap Between Fingers

$t_{m}$ : Thickness of the mirror

$x_{0}$ : Initial Overlap of the Fingers

$f_{d}$ : Width of the Fingers

When $V(t)$ is a periodic function, such as a sinusoid, the torque turns out to be a function of both time and angular displacement. Therefore, Eq. 1 becomes a nonlinear differential equation with time-varying coefficients. This implies that the resultant system is a parametric oscillator and will exhibit parametric resonances, and subharmonic oscillations.

Numerical simulations are performed using the model above and Matlab ${ }^{\odot}$ ODE solvers. Figure 3 shows that the behavior of the device when the input frequency is steadily increased or decreased is significantly different. Between the two jump frequencies, there are two stable solutions, and it is the initial conditions that determine which one is the actual solution that the oscillations will converge to. Therefore, for the regions, in which the nonlinear differential equation has two solutions, the simulation converges to the one with lower oscillation amplitude. For decreasing sweep simulations, the initial condition is larger than the maximum scan angle, and the simulation converged to the solution with larger oscillation amplitude.

Figure 4 illustrates the excitation and scan waveforms. A photodetector is placed at the center of the scan line and produces a short pulse each time the scanner goes through zero deflection angle. The square excitation waveform is at $2234 \mathrm{~Hz}$ and the zero crossings of the sinusoidal scan waveform are also at the same frequency. Since two peaks of the diode output correspond to one complete oscillation period, the scan waveform frequency is $1117 \mathrm{~Hz}$, half of the excitation frequency. Figure 4.b shows the sinusoidal scan waveform and the square wave excitation waveform, illustrating the subharmonic nature of the oscillations.

Figure 5 shows the simulation results for nonlinear frequency response characteristics and compares them with the experimental data. The graph is plotted for downsweep frequency response and three parametric resonances were captured. Numerical method successfully predicts the higher order parametric resonances and their associated jump frequencies. Numerical model is not as accurate in predicting the maximum scan amplitude. This is due to neglecting the fringing fields for the electrostatic torque calculations.

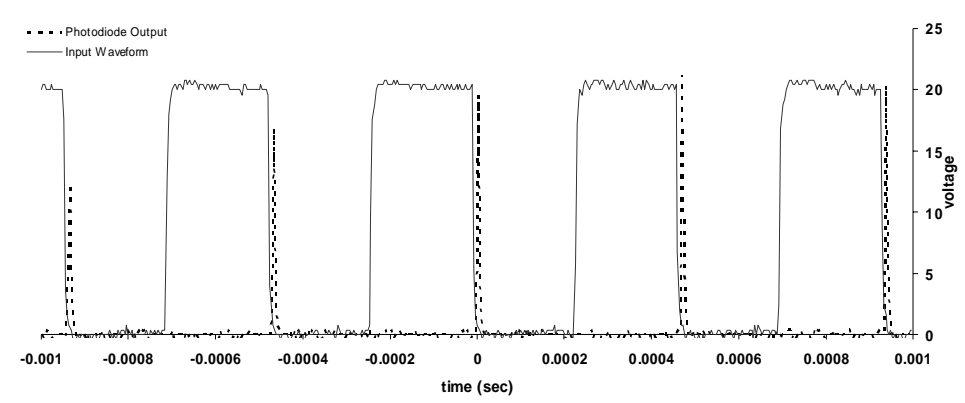

(a)

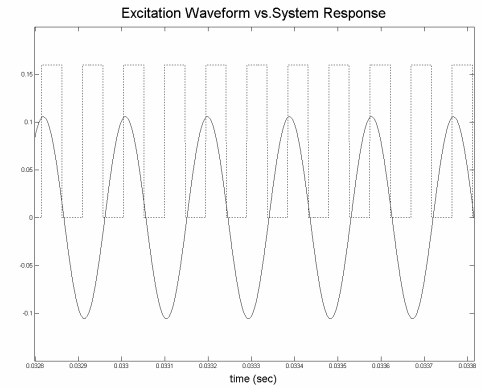

(b)

Figure 4: (a) Square wave excitation waveform (solid line) and photodiode output (dashed line) at the center of the scan line; (b) simulation result illustrating the sinusoidal scan waveform and the square excitation waveform 


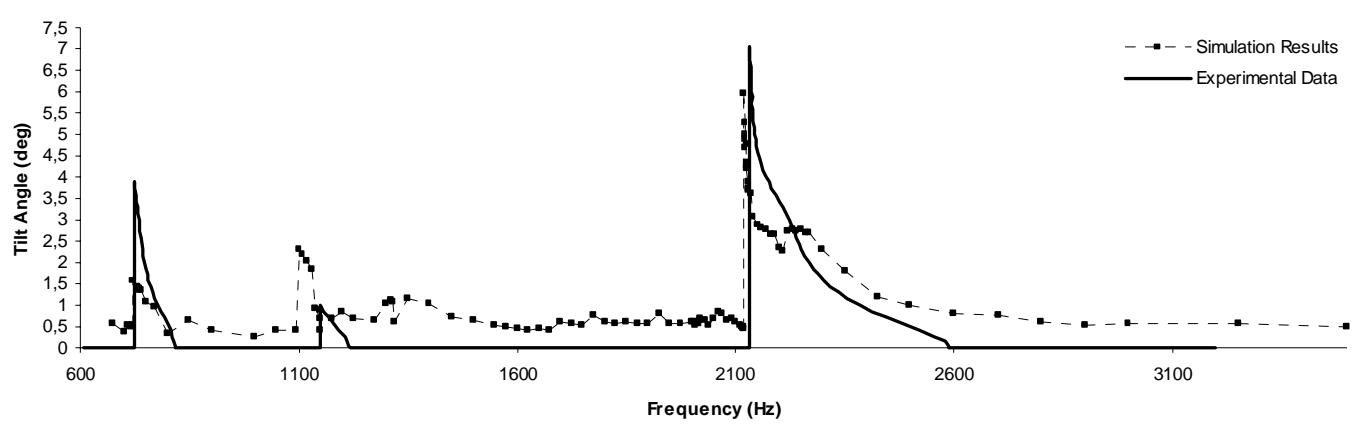

Figure 5: Comparison of the simulation and experimental results for the downsweep frequency response characteristics of the device for $20 \mathrm{~V}$ square wave excitation (shown in Figure 4.a)

\section{ANALYTICAL SOLUTION FOR $1^{\text {ST }}$ ORDER PARAMETRIC RESONANCE}

A general solution for the equation of motion of the microscanner is difficult to achieve. On the other hand, some reasonable assumptions can be made, in order to get some analytical expressions that will reveal some crucial characteristics of the solution. Assume that the input voltage waveform is the square root of a biased sinusoid function (this way, we can eliminate higher harmonics in the $V(t)^{2}$ term in Eq.2). In case of small amplitude oscillations, the torque function in Eq.3 can be simplified as:

$$
M(\theta, t)=-\left(r_{3} \theta^{3}+r_{1} \theta\right) A^{2}(\cos (w t)+1)
$$

In the above formula, rate of change of total capacitance is approximated with a third order polynomial. The torque function is odd; therefore no even order terms exist. Near the first parametric resonance, oscillation frequency is half of the drive frequency. Using this fact, and the simplified torque expression of Eq.4, Eq.3 can be rewritten for the first parametric resonance region as follows ${ }^{10}$ :

$$
\frac{d^{2} \theta}{d \tau^{2}}+\alpha \frac{d \theta}{d \tau}+(\beta+2 \delta \cos (2 \tau)) \theta+\left(\delta_{3}+\delta_{3} \cos (2 \tau)\right) \theta^{3}=0
$$

where,

$$
\tau=\frac{w t}{2}, \quad \alpha=\frac{2 b}{w I_{m}}, \quad \beta=4 \frac{\left(r_{1} A^{2}+K_{f}\right)}{w^{2} I_{m}}, \quad \delta=\frac{2 r_{1} A^{2}}{w^{2} I_{m}}, \quad \delta_{3}=4 \frac{r_{3} A^{2}}{w^{2} I_{m}}
$$

If the damping is small in the system, only $\beta$ is in the order of 1 , whereas the other terms are at least one order of magnitude smaller than $\beta$. Therefore, perturbation methods may be utilized in the solution of Eq. 5. If we define the perturbation parameter $\varepsilon$ as equal to $\delta$, Eq.5 becomes a perturbed Mathieu equation and it can be solved using method of two-variable expansion ${ }^{9}$. This method is based on the fact that possible solution of Eq. 5 includes two different timescales. One of those time scales is close to $\tau$ and defines the period of the motion. The other time scale, on the other hand, is much slower and modulates the amplitude of the oscillations ${ }^{10}$. If the time scales are defined as $\tau$ and $\eta=\varepsilon t$, and we assume that $\theta=\theta_{0}+\varepsilon \theta_{1}, \beta=1+\delta \beta_{1}$, the solution of Eq.5 is given as follows:

$$
\theta=A(\tau) \cos \left(\frac{1}{2} \xi\right)+B(\tau) \cos \left(\frac{1}{2} \xi\right)
$$

where $\mathrm{A}$ and $\mathrm{B}$ are given as the solution of following coupled differential equations: 


$$
\begin{aligned}
& \frac{d A}{d \tau}=-\frac{\mu}{2} B+\frac{B}{2}\left(\beta_{1}-1\right)+\frac{B \delta_{3}}{8 \varepsilon}\left(3 A^{2}+B^{2}\right) \\
& \frac{d B}{d \tau}=-\frac{\mu}{2} A-\frac{A}{2}\left(\beta_{1}+1\right)-\frac{A \delta_{3}}{8 \varepsilon}\left(5 A^{2}+B^{2}\right)
\end{aligned}
$$

Limits of the bistable region on the $\beta-\delta$ and the map of this region on the voltage-frequency plane are shown in Figure 6. On figure 6a, the frequency range between the arms of the plot at a certain voltage corresponds to the frequency range between the two jump frequencies on Figure 3. Analytical model can successfully estimate the peak frequency for the stability region; however, the deviation from the model in higher voltage values is evident. This is because that for at atmospheric pressure, the damping term in Eq.5 is not small enough to be assumed as a perturbation in the system. In vacuum environment, the model is predicted to give much accurate results. Vacuum measurements will be performed as future work.

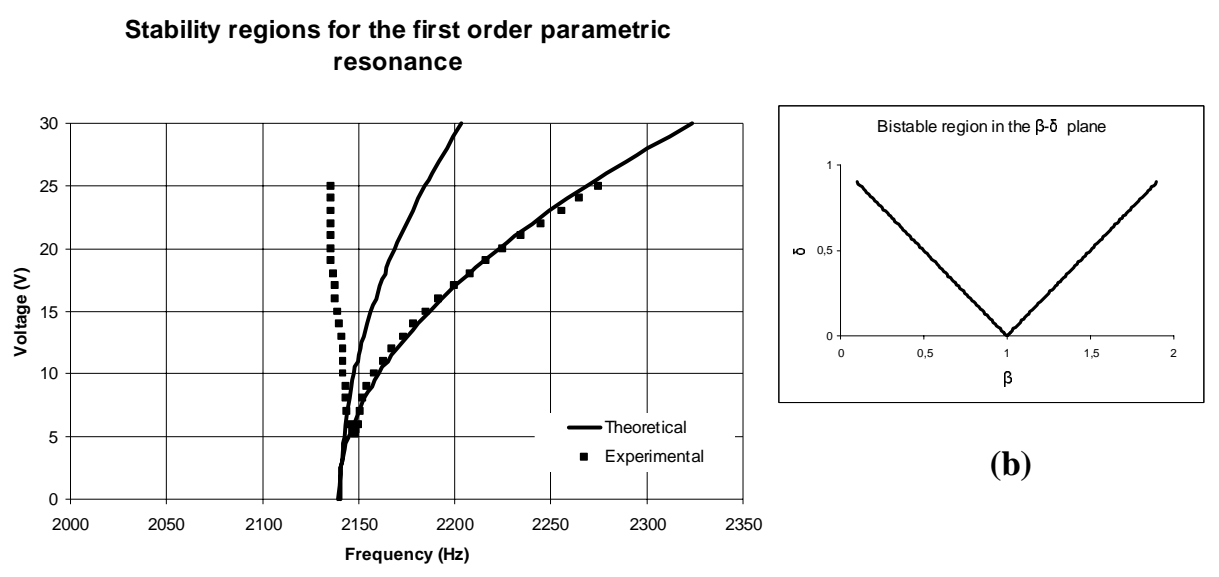

(a)

Figure 6: Analytical and experimental results for the stability region a) on the voltage-frequency plane, b) on the $\beta-\delta$ plane. Theoretical plots are drawn assuming that there is no damping in the system. However actual device is operated in the atmospheric pressure and significant damping is present in the system. Therefore, the tip of the stability curve is lifted upwards and the curve is widened considerably.

The theoretical plots assume that there is no damping in the system; therefore the minimum points are on the $\mathrm{x}$-axis. However, the experimental data are taken in atmospheric pressure and there is significant air damping in the system $(\mathrm{Q}$ factor of the system is only 22). Damping lifts the tongue upwards and smoothes the tip of the stability curve. Analytically, the tip of the curve is found to be at $2242 \mathrm{~Hz}$. The actual value is $2248 \mathrm{~Hz}$, which is in close agreement with the analytical result.

\section{HIGHER ORDER PARAMETRIC RESONANCES}

In addition to the first order parametric resonance that we have analyzed so far, parametric systems have more resonances located near $2 f_{r} / n$, where $f_{r}$ is the mechanical resonance frequency of the system, and $n$ is any positive integer greater than one. As shown in the analytical analysis, as the damping in the system is increased, the threshold voltage required for observing the parametric resonances rises. Due to damping, it is difficult to observe higher order parametric resonances at atmospheric pressure for most MEMS devices. However, the device in this work gives rise to higher order parametric resonances even at atmospheric pressure. Figure 7 shows the experimental frequency response curves for the microscanner with both square and sinusoidal excitation. With square wave excitation, it is possible to observe 8 different orders of parametric resonances, whereas with sinusoidal excitation, number of observable parametric resonances is only two. 


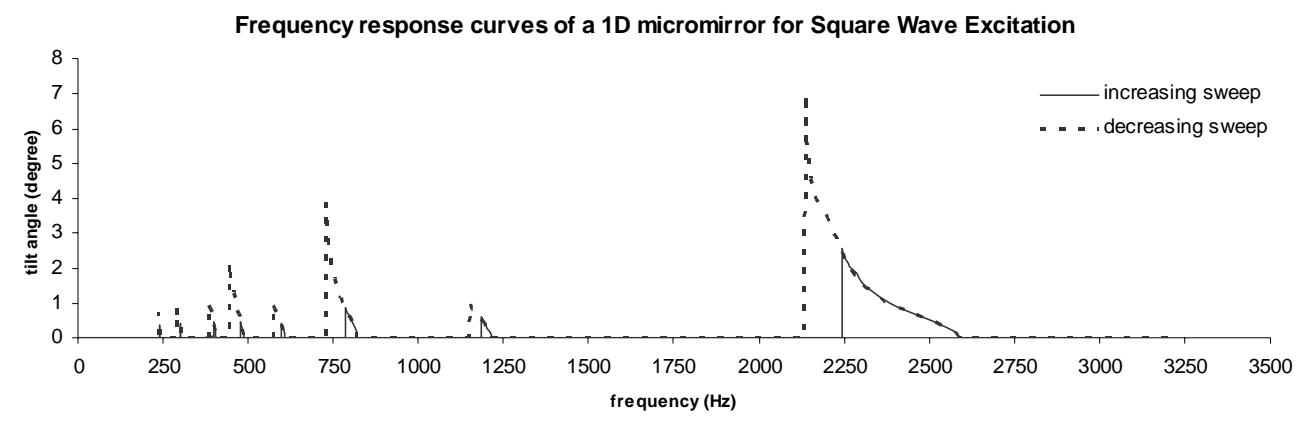

(a)

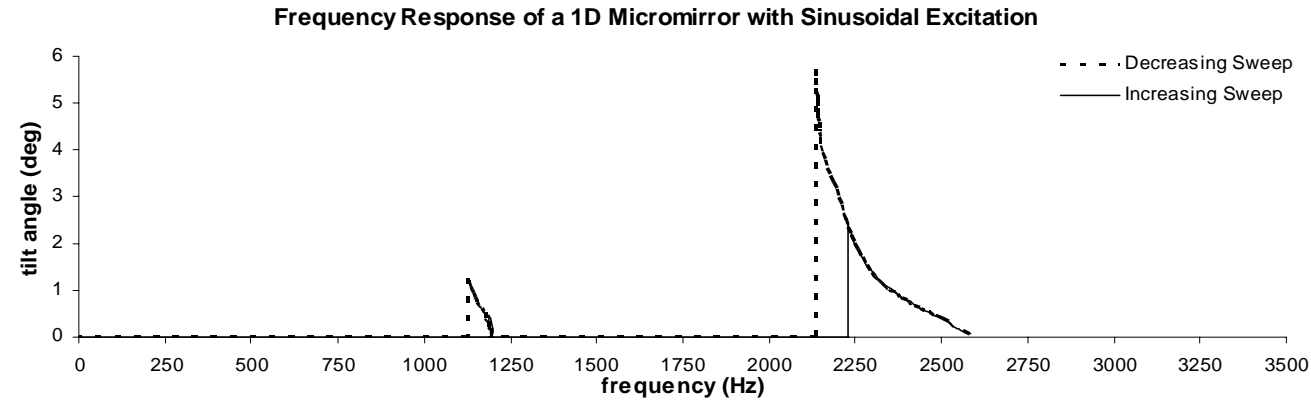

(b)

Figure 7: Higher order parametric resonances a) with $20 \mathrm{~V}$ square wave input, b) $20 \mathrm{~V}$ sine wave input

For square wave input, the response is not a pure sinusoid for high order resonances. Scan wave shows an unusual behavior and changes oscillation frequency and amplitude at each half cycle of the square wave. When excitation is in the OFF state (meaning that force is not present in the system), the system makes free oscillation at its natural resonant frequency, when the excitation is in the ON state, the mirror oscillates at a higher frequency, which can be calculated using the excitation and natural frequencies. Figure 8 shows the experimental results illustrating this behavior. Such behavior is observed in very high order resonances. In Figure 9 simulated waveforms for varying frequency behavior can be seen.

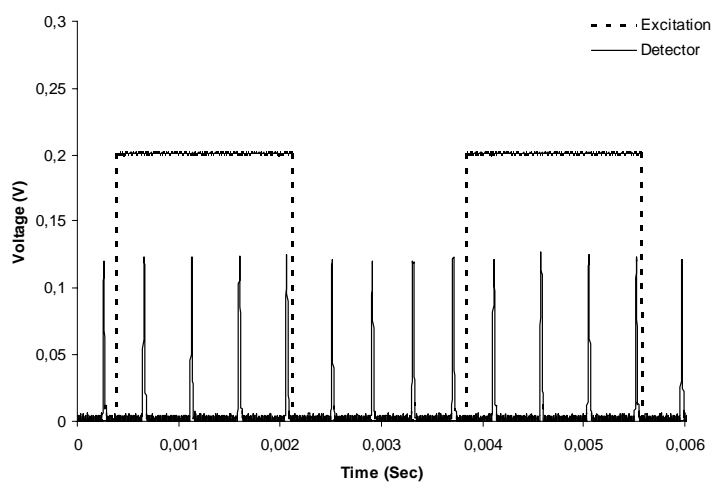

(a)

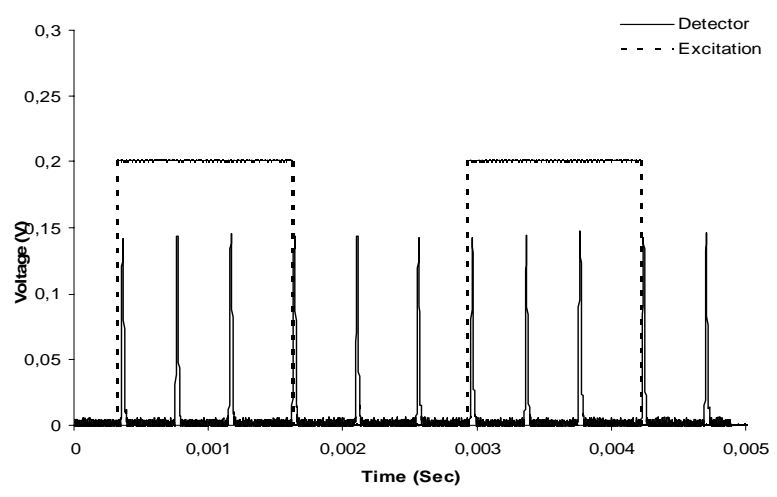

(b)

Figure 8: Varying frequency oscillations (a) At $7^{\text {th }}$ order parametric resonance and $289 \mathrm{~Hz}$, (b) At $6^{\text {th }}$ order parametric resonance and $384 \mathrm{~Hz}$. 


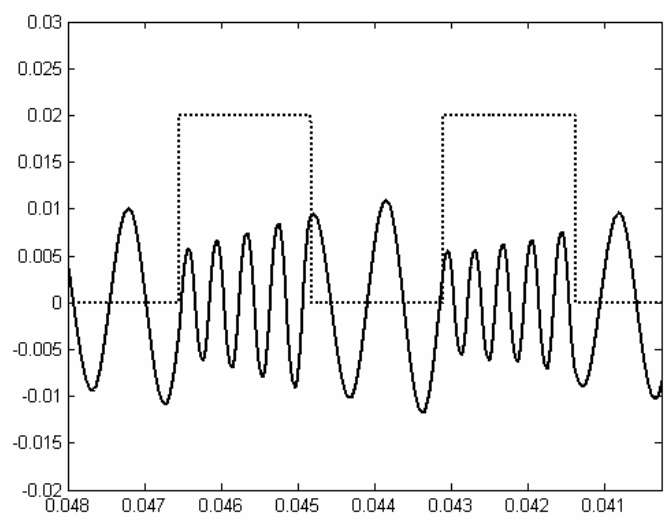

Figure 9: Simulation results for the varying frequency behavior. The simulation was done at $380 \mathrm{~Hz}$, for $20 \mathrm{~V}$ square wave input.

\section{DISCUSSION AND FUTURE WORK}

We have presented an analytical and a numerical model for a comb-driven microscanner. The numerical model can be utilized up to 20 degrees of angular displacement (the limit is the validity of small angle approximation) and predicts higher order parametric resonances, as well as the fundamental resonance. The significance of the model is within its ability to simulate high deflection angles, which are common in display and scanning applications. The analytical model is valid for a simplified excitation waveform, small angular displacements, and negligible damping. Analysis in this paper is done for the fundamental resonance; however similar methods can be used to reach approximate analytical solutions for higher order resonances. Analytical results will be extended to higher order resonances in future work. The analytical model cannot predict the instability region of the voltage-frequency curve very accurately. This is primarily, due to the loose satisfaction of the low damping assumption at atmospheric pressure. Vacuum testing of the devices will be carried out as a next step, in order to obtain better agreement between the analytical model and the experimental results.

\section{ACKNOWLEDGEMENTS}

We'd like to thank Dr.Wyatt Davis (Microvision Inc) and Dr.Alexander Wolter (Fraunhofer IPMS) for helpful discussions

\section{REFERENCES}

1. S. Rodgers, S. Kota, J. Hetrick, Z. Li, B. Jensen, T. Krygowski, S. Miller, S. Barnes, M. Burg, "A new class of high force, low-voltage, compliant actuation systems," Sensors \& Actuators Workshop, 2000

2. H. Schenk, P. Dürr, D. Kunze, H. Lakner, H. Kück, "A resonantly excited 2D micro-scanning-mirror with large deflection," Sensors and Actuators A, Vol. 89, p.104-111 (2001).

3. P. R. Patterson, D. Hah, H. Nguyen, H. Toshiyoshi, R. Chao, M. Wu, "A scanning micromirror with angular comb-drive actuation," International Conference on Micro Electro Mechanical Systems, Proc. IEEE, p.544-547 (2002).

4. L. Lin, R. T. Howe, A. P. Pisano, "Micromechanical filters for signal processing," Journal of Microelectromechanical Systems, Vol. 7, No. 3, p.286-294 (1998).

5. C. T. C. Nguyen, "High-Q micromechanical oscillators and filters for communications," International Symposium on Circuits and Systems, Proc. IEEE, p.2825-2828 (1997).

6. J. D. Grade, K. Y. Yasumura, H. Jerman, "A DRIE comb-drive actuator with large, stable deflection range for use as an optical shutter, "International Conference on Solid-State Sensors, Actuators and Microsystems, Proc. IEEE/EDS, Boston, Massachusetts, USA (2003). 
7. L. Zhou, M. Last, V. Milanovic, J. M. Kahn, K. S. J. Pister, "Two-axis scanning mirror for free-space optical communication between UAVs, ” International Conference on Optical MEMS and Their Applications, Proc. IEEE/LEOS, p.1-2, Hawaii, USA (2003).

8. Y. Mitropolskii, N. V. Dao, "Applied Asymptotic Methods in nonlinear oscillations", Kluwe Academic Publishers, Boston (1997).

9. R. H. Rand, “Lecture Notes on Nonlinear Vibrations, ” Version 34a, Available online at http://www.tam.cornell.edu/randdocs, (2000).

10. W. Zhang, R. Baskaran, K. L. Turner, "Effect of cubic nonlinearity on auto-parametrically amplified resonant MEMS mass sensor,” Sensors and Actuators A, Vol. 102, p.139-150 (2002).

11. K. L. Turner, S. A. Miller, P. G. Hartwells, N. C. MacDonald, S. H. Strogatz, "Five parametric resonances in a microelectromechanical system,” Nature, Vol. 396, p.149-152 (1998).

12. H. Shenk, P. Dürr, T. Haase, D. Kunze, U. Sobe, H. Lakner, H. Kück, "Large deflection micromechanical scanning mirrors for linear scans and pattern generation,” Journal of Selected Topics in Quantum Electronics, Vol. 6, No. 5, p.715-722 (2000).

13. H. Schenk, A. Wolter, H. Lakner, "Design optimization of an electrostatically driven micro scanning mirror," Conf. on MOEMS and Miniaturized Systems, Proc. SPIE, Vol. 4561, p.35-44, San Francisco, Calif., USA, (2001)

14. J. Chen, S. Kang, “Dynamic macromodeling of MEMS mirror devices, ” International Electron Device Meeting, Proc. IEEE, p.41.5.1-41.5.4, Washington DC, USA, (2001). 